\title{
Epidemiology and Evolution of Antiretroviral Drug Resistance
} Mark A Wainberg*‡

\author{
Address: McGill University AIDS Centre, Jewish General Hospital, Montreal, Quebec, CANADA \\ Email: Mark A Wainberg* - mark.wainberg@mcgill.ca \\ * Corresponding author $\ddagger$ Presenting author
}

from 2005 International Meeting of The Institute of Human Virology

Baltimore, USA, 29 August - 2 September 2005

Published: 8 December 2005

Retrovirology 2005, 2(SuppI I):SI 22 doi: I0. I I86/I742-4690-2-SI-SI 22

The development of HIV drug resistance is a formidable obstacle in the long term success of antiretroviral therapeutic regimens. To date, resistance has occurred against all drugs that are in widespread use for treatment of HIV disease. Moreover, HIV drug resistant viruses can be sexually transmitted and it is now estimated that as many as $10 \%$ of new HIV infections in Western countries may carry at least one mutation associated with HIV drug resistance. In some cases, newly infected individuals may even harbour viruses that are resistant to two or even three classes of antiretroviral drugs. Studies have shown that mutations which are transmitted from one individual to another may often persist over long periods of time i.e. 27 years. Although many mutations may also revert to wild-type, a danger is that they will have become permanently archived in a patient's long-lived memory Tcells, and that this may preclude future therapeutic options.

At the same time, many of the mutations associated with HIV drug resistance may cause diminished "replicative capacity" and, indeed, some clinical studies have shown that at least some individuals infected with multi-drug resistant strains may have lower viral loads over periods of several years than do individuals infected with wild-type strains. It is also interesting that some of the mutations associated with HIV drug resistance may be less easily transmitted than others or are present at diminished frequency in newly infected hosts. In general, it appears as though thymidine analogue mutations (TAMs), associated with resistance to zidovudine and stavudine, as well as mutations associated with non-nucleoside reverse transcriptase inhibitors (NNRTIs), may be transmitted fairly efficiently while mutations associated with resistance to other nucleosides, e.g. 3TC, TDF (M184V and K65R), may be transmitted much less frequently.
This subject has relevance in view of the widespread use of co-formulated nevirapine/3TC/stavudine (TRImune) as a first line regimen favored by the World Health Organisation (WHO) for use in many developing countries. There is a strong possibility that resistance might develop over time against several of the drugs in this regimen that have low genetic barriers for resistance, meaning that only a single point mutation in the reverse transcriptase gene may yield significantly diminished levels of antiviral activity (3TC and NVP). Nonetheless, we should support this WHO initiative $(3 \times 5)$ because this regimen is likely to have the greatest impact and save millions of lives during the next several years and, as well, will impact on rates of HIV transmission. 